Yanira Olivares Ramírez

Universidad de Santiago de Chile

yeolivares@uc.cl

\title{
Representaciones de la violencia contra la mujer en la prensa chilena ${ }^{1}$
}

\author{
Representations of violence against women in the chilean press
}

\section{Resumen}

Este artículo se centra en los encuadres que los medios de comunicación le otorgan a sucesos de violencia contra la mujer en Chile. Para ello, la investigación ofrece un análisis de contenido a la prensa escrita chilena, focalizándose en el tratamiento informativo y la cobertura mediática otorgada al feminicidio frustrado en contra de Nabila Rifo, hecho ocurrido el año 2016.

Se observó una gran cobertura y relevancia al caso, lo que sugiere un posicionamiento de la violencia contra la mujer en la agenda mediática chilena. No obstante, el tratamiento informativo demostró diversas falencias (rupturas epistemológicas, ausencia de contexto, sobreexposición de la víctima, móviles y justificaciones, exigua incorporación de fuentes especializadas en el tema, escasa información educativa, encuadres morbosos, entre otros).

En consecuencia, se propone que, a pesar de la visibilización del tema, el tratamiento informativo en la prensa chilena utiliza enfoques que no incorporan una perspectiva de género, facilitando un segundo espacio de violencia para la víctima.

Palabras clave: Violencia contra las mujeres, victimización secundaria, encuadre.

\begin{abstract}
This article focuses on the frames that the media give to events of violence against women in Chile. For this, the research offers a content analysis to the Chilean press, focusing on the information treatment and media coverage given to the frustrated feminicide against Nabila Rifo, fact occurred in 2016.
\end{abstract}

\footnotetext{
${ }^{1}$ Este artículo presenta una actualización de publicaciones académicas efectuada en el marco de una tesis de Magíster en Ciencias de la Comunicación.
} 
Yanira Olivares Ramírez.

A great coverage and relevance to the case was observed, which suggests a positioning of violence against women in the Chilean media agenda. However, the informative treatment showed several shortcomings (epistemological ruptures, absence of context, overexposure of the victim, motives and justifications, poor incorporation of specialized sources in the subject, scarce educational information, morbid frames, among others).

Consequently, it is proposed that, despite the visibility of the issue, the information treatment in the Chilean press uses approaches that do not incorporate a gender perspective, facilitating a second space of violence for the victim.

Keywords: Violence against women, secondary victimization, frame.

\section{Introducción}

"Los crímenes contra las mujeres

no son crímenes de odio

sino que de orden político”

Rita Segato

El conjunto de características diferenciadas que cada sociedad asigna a los hombres y a las mujeres estaría marcada por relaciones de poder (Scott, 1996), donde la mujer sería relegada a una situación de inferioridad (Beauvoir, 1949). Desde esta perspectiva el género marcaría cierto prestigio al interior de una sociedad, existiendo una desvalorización de lo femenino frente a lo masculino, donde el factor de riesgo o de vulnerabilidad es el solo hecho de ser mujer (Rico, 1996).

Por ello, a lo largo de la historia de la humanidad las mujeres han tenido que luchar constantemente para hacer valer sus derechos. No obstante, en la actualidad, a pesar de los múltiples esfuerzos y avances que se han hecho en materia de género, el contexto no es favorable. Esto se debe a que la violencia contra la mujer (VCM), desde su dimensión simbólica, resulta ser un hábito invisible para la sociedad, lo que impide identificarla, a tal punto que ni siquiera las instituciones, ni las víctimas son capaces de percibirlas (Bordieu, 2000).

Frente a esta preocupante situación, los medios de comunicación (MCM) pueden cumplir un rol fundamental, principalmente visibilizando e informando sobre la violencia contra la mujer.

La teoría del framing y la agenda setting, proponen que los media seleccionan los aspectos de una realidad, les dan orden y relevancia en un texto. Por ello, se entiende el frame 


\section{Yanira Olivares Ramírez.}

como "principios organizadores socialmente compartidos y persistentes en el tiempo, que trabajan simbólicamente para estructurar el mundo social de modo significativo" (Reese en Aruguete, 2011:71). Así, además de informar, los medios de comunicación son capaces de producir y expandir el conocimiento, sugerir cuál es el tema relevante mediante su énfasis o exclusión, formar opinión y configurar el espacio público, siendo los encargados de definir nuestro mundo (Lippmann, 1922).

Actualmente, en las sociedades modernas ya no es necesario compartir un lugar en común para participar de lo público. Ya que los media tienen la capacidad de renovar el vínculo de propiedad pública al presentar los diferentes contenidos de la vida social a la ciudadanía, configurando un nuevo espacio público. Lo que sin duda les delega una gran responsabilidad para el cultivo del destino colectivo, al ser la principal fuente de información y de producción de formas simbólicas que sostienen y constituyen a la sociedad (Thompson, 1998).

Sin embargo, desde una perspectiva de género, las cifras y los estudios respecto a los medios de comunicación, tanto en Chile como a nivel mundial demuestran que:

a.-Los medios de comunicación son sexistas y machistas

El Global Report on the status of women the News Media (2011) arrojó que en las compañías de comunicación tan solo un 33,8\% de los miembros son mujeres. Además de esas pocas féminas, la gran mayoría desempeña labores como reporteras, áreas de administración, ventas y finanzas, cargos que -como lo menciona la investigación- son normalmente ocupados por mujeres.

Asimismo, las mujeres en altos cargos son prácticamente ausentes, por ejemplo un 8,6\% tiene puestos en gobernabilidad y un 7,3\% en alta gerencia. Un claro ejemplo es Televisión Nacional de Chile, donde de los 20 editores solo 2 son mujeres (OGE, 2016). En la misma línea, el estudio de la Federación Internacional de Periodistas (FIP, 2017) señala que un $48 \%$ de las periodistas ha sufrido violencia de género en el trabajo.

b.- Medios y periodistas sin perspectiva de género

El Global Media Monitoring Project (2010) luego de hacer análisis de contenido a más de 100 países -incluido Chile- reveló que el 46\% de las noticias (impresas y TV) promueven estereotipos de género, asimismo agrega que a nivel global solo un $6 \%$ de las noticias promueve igualdad de género. Por otra parte el Global Report on the status of women the News Media 


\section{Yanira Olivares Ramírez.}

(2011) arrojó que, particularmente en Chile, de los medios analizados solo un $11 \%$ tomo una postura de género.

Fabiola Gutiérrez, Presidenta de la Comisión de Género del Colegio de Periodistas, en el conversatorio "Medios de comunicación sexismo y transformaciones culturales" (2018), planteó como uno de los desafíos actuales, reflexionar sobre la formación del conglomerado, considerando que uno de los elementos claves debe ser reeducar a los periodistas e incluir en las mallas curriculares cursos de comunicación con enfoque de género, ya que no existen.

En la misma línea, la revisión literaria muestra antecedentes que permiten afirmar que la prensa chilena reproduce un imaginario machista y patriarcal, colaborando activamente en la construcción de un modelo único de la mujer, asociándolas a estereotipos de antaño (hogar, familia, sexualidad). Elementos que también se ven presentes al momento de tratar noticias sobre feminicidios, produciendo enfoques y tratamientos erróneos en la cobertura de estos casos. Cometiendo errores recurrentes como violar la intimidad de las víctimas, entregar distinto trato según estrato social, sensacionalismo, justificación del agresor por medio de patologías, minimizar la agresión, ausencia de empatía con la víctima, conceptos erróneos, falta de contextualización y promover el morbo (Morales y González, 2007; Lagunas y Lencina, 2010; Pérez, 2010; Angélico, Fischberg y Maffeo, 2014; Ananías y Vergara, 2016).

De este modo, los medios de comunicación se han convertido en instituciones que participan de la violencia, justificándola y reproduciéndola, lo que se ha denominado "consentimiento silencioso" (Ramos, en Jiménez, 2005: 38). Y, al mismo tiempo -en algunos casos- genera un segundo espacio de violencia, lo que ha sido denominado victimización secundaría.

A partir de este contexto, surge el interés por actualizar el panorama general de los medios de comunicación en Chile respecto a temas de género. Por ello, el presente artículo ofrece una revisión con perspectiva de género sobre el tratamiento y la cobertura que la prensa chilena le otorga a casos de violencia contra la mujer. El análisis se centra en el caso de Nabila Rifo, un feminicidio frustrado cometido por Mauricio Ortega, el año 2016 en la ciudad de Coyhaique, suceso que generó gran conmoción en el país y tuvo una gran cobertura mediática.

El estudio realizado es de carcater descriptivo, con un enfoque mixto vinculando datos cuantitativos y cualitativos. La técnica escogida para realizar el levantamiento de datos es el 


\section{Yanira Olivares Ramírez.}

análisis de contenido, ya que permite analizar, clasificar y codificar mensajes al interior de un corpus determinado. Además, "procura comprender los datos, no solo como un conjunto de acontecimientos físicos sino como fenómenos simbólicos" (Krippendorff, 1990:7).

Para codificar la información se utilizó una ficha de análisis compuesta por cinco categorías $^{2}$, la que fue aplicada a una muestra total de 89 noticias sobre el caso de Nabila Rifo. Los ejemplares analizados fueron publicados durante periodos claves entre mayo del año $2016 \mathrm{y}$ julio de 2017 y pertenecen a los diarios La Cuarta, La Tercera, La Hora, El Mercurio, La Segunda, HoyxHoy, Las Últimas Noticias y Publimetro ${ }^{3}$

\section{Desarrollo: Hallazgos sobre el tratamiento y cobertura de la prensa chilena al caso de Nabila Rifo}

\section{Hitos e interpretaciones}

Teniendo en cuenta la amplitud del caso, el análisis se delimitó a cuatro hitos relevantes. Además, con el fin de evidenciar si el tratamiento informativo coloca énfasis en momentos positivos o negativos para la víctima se realiza una propuesta interpretativa de cada suceso. De este modo, cada hito será categorizado bajo la premisa de: favorable, desfavorable o neutro en relación a Nabila Rifo.

HITO 1: Ocurre el crimen contra Nabila Rifo

MAYO 14, 2016

Desfavorable

El Hito 1 se considera desfavorable, puesto que es la primera aparición mediática de Nabila Rifo, en donde los medios presentan a la mujer bajo la figura de "víctima de una brutal agresión”. En este suceso las informaciones se concentran en entregar detalles de la víctima, y

\footnotetext{
${ }^{2}$ Categorías de análisis: datos generales, ubicación en el medio y extensión, contenido, lenguaje y fuentes.

${ }^{3}$ La elección de estos diarios se sustentó en los estudios de la Asociación Chilena de Agencia de Medios (AAM), quienes los reconocen como diarios consolidados, de gran difusión a nivel nacional y con altos niveles de audiencia al interior de la sociedad chilena.
} 


\section{Yanira Olivares Ramírez.}

colocar énfasis en la extracción de sus ojos, reiterando su riesgo vital y el desconocimiento sobre el responsable de los hechos.

HITO 2: Nabila Rifo declara ante tribunales

MARZO 23, 2017

Neutro

En este hito, se muestra a Nabila declarando por primera vez en el tribunal oral. Inicialmente se consideró como un hecho desfavorable, ya que la víctima debe recordar los hechos de violencia y, al mismo tiempo exponerse a diversos cuestionamientos durante el juicio. Es más, tras su declaración y las interpelaciones del abogado de su agresor, Nabila reconoce que: "Me han faltado el respeto, pero yo sé cómo soy, sé cómo he sido y me valoro. Lo demás no me interesa" (La Tercera, 25/03/2017).

No obstante, es posible observar que también es una instancia favorable, ya que por primera vez se obtiene la versión de ella. En la declaración entrega detalles del suceso y culpa como único responsable a Mauricio Ortega y, al mismo tiempo utiliza este espacio para contar sobre su nueva vida, libre de violencia, haciendo un llamado a las agredidas a denunciar sobre los maltratos. Hecho que fue valorado en los medios de comunicación y por algunos líderes de opinión ("Tras el horror que vivió y vive Nabila Rifo, destaco la valentía de esta mujer para entregar su testimonio" (La Cuarta, 24/04/2017). Por estas razones fue complejo categorizarlo exclusivamente como favorable o desfavorable, categorizándolo como un hito neutro.

\section{HITO 3: Veredicto}

ABRIL 18, 2017

Favorable

Este hito corresponde al veredicto del juicio, en donde se entregó un fallo a favor de Nabila Rifo, indicando como único culpable a Mauricio Ortega. Sujeto a quien se le otorga una condena de 26 años, este hecho deja conforme a la víctima quien aseguró "que el veredicto contra Ortega le entregó más alivio" (La Cuarta, 19/04/2017). De este modo, el suceso informativo muestra a la víctima satisfecha ante el fallo, siendo un hito favorable. 


\section{Yanira Olivares Ramírez.}

HITO 4: Rebaja de condena para Mauricio Ortega

JULIO 11, 2017

Desfavorable

Por otra parte, en el Hito 4, la Corte Suprema rebaja la sentencia de 26 a 18 años tras eliminar el crimen de femicidio frustrado. Hecho que suscitó un gran rechazo y descontento en la ciudadanía, organizaciones y evidentemente en Nabila Rifo. Ante los hechos Nabila reconoce que: "No lo creía, dije ¿cómo puede ser posible? Escuché al juez hablar y me puse a llorar, porque no podía creerlo" (La Tercera, 13/07/2017). De este modo, es un suceso desfavorable para la víctima, ya que reduce los años de cárcel para su victimario, es decir favorece al agresor.

\section{Ubicación en el medio y extensión}

Tabla 1. Resultados ubicación en el medio y extensión

\begin{tabular}{|l|c|c|c|c|c|}
\hline \multicolumn{7}{|c|}{ CASO NABILA RIFO } \\
\hline HITOS & HITO & HITO & HITO & HITO & TOTAL \\
& 1 & 2 & 3 & 4 & \\
\hline Cantidad de noticias & 28 & 20 & 19 & 22 & 89 \\
\hline Referencia en portada & 8 & 7 & 10 & 12 & 37 \\
\hline Material gráfico en portada & 5 & 6 & 7 & 4 & 22 \\
\hline Pertenece a la víctima & 1 & 6 & 5 & 1 & 13 \\
\hline Pertenece al agresor & 3 & 0 & 4 & 2 & 9 \\
\hline Espacio que ocupa noticia & $55 \%$ & $49 \%$ & $63 \%$ & $61 \%$ & $57 \%$ \\
interior & & & & & \\
\hline
\end{tabular}


Yanira Olivares Ramírez.

\begin{tabular}{|c|c|c|c|c|c|}
\hline Página entre $1-10$ & 17 & 13 & 14 & 15 & 59 \\
\hline Página entre $11-20$ & 9 & 4 & 4 & 7 & 24 \\
\hline Página entre 21-30 & 3 & 7 & 1 & 2 & 13 \\
\hline Página entre $31-40$ & 0 & 0 & 0 & 1 & 1 \\
\hline Página entre 41-50 & 0 & 0 & 1 & 0 & 1 \\
\hline Página izquierda & 19 & 17 & 15 & 18 & 69 \\
\hline Página derecha & 10 & 7 & 5 & 7 & 29 \\
\hline Autora mujer & 11 & 1 & 9 & 5 & 26 \\
\hline Autor hombre & 5 & 3 & 8 & 5 & 21 \\
\hline Iniciales & 5 & 6 & 4 & 3 & 18 \\
\hline Firma diario & 0 & 0 & 0 & 1 & 1 \\
\hline Firma agencia & 4 & 1 & 0 & 2 & 7 \\
\hline Sin autor & 6 & 9 & 1 & 6 & 22 \\
\hline
\end{tabular}

Fuente: Elaboración propia.

\section{Cobertura}

A partir de los datos obtenidos, se demuestra que la prensa entregó una cobertura informativa temporalmente extensa (dos años), una alta frecuencia en la cantidad de apariciones (89) y una gran relevancia a la información. 


\section{Yanira Olivares Ramírez.}

La importancia del contenido informativo se sustenta en la disposición, acompañamiento de material visual y extensión de la pieza periodística al interior del diario ${ }^{4}$. A partir de las 89 noticias, se constató que el $42 \%$ de ellas tuvo aparición en portada, y el 59\% incorporaba material gráfico. Asimismo $60 \%$ de las noticias fueron ubicadas entre las diez primeras páginas, $71 \%$ estaban dispuestas en la página izquierda del diario, el promedio de extensión fue de un 57\%. De este modo se constata que la agenda mediática de la prensa chilena posicionó el caso de Nabila Rifo, un hecho de violencia contra la mujer, como un suceso relevante para el acontecer nacional.

\section{Hitos de mayor y menor relevancia}

A partir de la cantidad de noticias, es posible evidenciar que los hitos con mayor cantidad de noticias al interior de la prensa chilena, son sucesos desfavorables para la víctima, es decir, Hito 1-Ocurre el crimen (28) e Hito 4-Rebaja de Condena (22). En oposición, el hito con menor cantidad de noticias corresponde al único suceso favorable para la víctima, Hito 3-Veredicto (19). Es más, también fue el único periodo donde se encontró diarios con 0 publicaciones de noticias sobre el caso (Publimetro y La Segunda).

Esta práctica periodística y tipo de encuadre, coloca mayor énfasis y relevancia en aquellos contextos desfavorables para las mujeres, lo que fomenta la representación estereotipada de la mujer víctima, mujer vulnerable.

\section{Diferencias en la cobertura por grupos editoriales}

\section{Tabla 2. Cantidad de noticias agrupadas por interpretación de hitos}

\footnotetext{
${ }^{4}$ Siguiendo los índices de atención propuestos por diversos autores: la aparición en portada, el acompañamiento de material visual, la ubicación en páginas impar, la disposición entre las primeras páginas, sus grandes dimensiones, la presencia de entradilla y la huella de autoría aumentan su importancia y visibilidad, denotando de este modo la jerarquía que el medio le otorga a un caso (Budd, 1964; McCombs y Shaw, 1972; Weaver, Graber, McCombs \& Eyal, 1977; Casermeiro de Pereson, 2004; Gutiérrez Coba, 2001; Igartua, Muñiz \& Cheng, 2005; Aruguete, 2009; Odriozola Chené, 2012).
} 
Yanira Olivares Ramírez.

\begin{tabular}{|c|c|c|c|c|}
\hline EDITORIAL & DIARIO & $\begin{array}{c}\text { DESFAVORABLES } \\
\text { HITO 1-HITO } 4\end{array}$ & $\begin{array}{c}\text { NEUTRAS Y } \\
\text { FAVORABLES } \\
\text { HITO } 2 \text { - HITO } 3\end{array}$ & TOTAL \\
\hline \multirow{3}{*}{$\begin{array}{l}\text { COPESA } \\
\text { (49 noticias) }\end{array}$} & LA CUARTA & 8 & 10 & 18 \\
\hline & $\begin{array}{l}\text { LA } \\
\text { TERCERA }\end{array}$ & 15 & 8 & 23 \\
\hline & LA HORA & 5 & 3 & 8 \\
\hline \multirow{4}{*}{$\begin{array}{l}\text { EL MERCURIO S.A. } \\
\text { (31 noticias) }\end{array}$} & $\begin{array}{l}\text { EL } \\
\text { MERCURIO }\end{array}$ & 2 & 7 & 9 \\
\hline & $\begin{array}{l}\text { LA } \\
\text { SEGUNDA }\end{array}$ & 2 & 2 & 4 \\
\hline & HOYXHOY & 9 & 2 & 11 \\
\hline & LUN & 2 & 5 & 7 \\
\hline $\begin{array}{l}\text { METRO } \\
\text { INTERNATIONAL } \\
\text { (9 noticias) }\end{array}$ & $\begin{array}{l}\text { PUBLIMETR } \\
\text { O }\end{array}$ & 7 & 2 & 9 \\
\hline
\end{tabular}

Fuente: Elaboración propia

El grupo editorial Copesa, lideró en cantidad de noticias. En otras palabras, es la corporación que entregó mayor visibilidad a un caso de violencia contra la mujer. No obstante, como se observa en la tabla, al agrupar las noticias desfavorables (Hito 1, Hito 4) versus las noticias neutras y favorables (Hito 2, Hito 3), los medios que dieron mayor énfasis y cobertura a los sucesos desfavorables son: La Tercera, La Hora, HoyxHoy y el diario Publimetro. 


\section{Yanira Olivares Ramírez.}

Estos diarios pertenecen a los tres grupos editoriales analizados, siendo posible inferir que no existe un lineamiento o consenso a nivel editorial al momento de entregar mayor o menor cobertura a los contextos favorables o desfavorables para las víctimas de violencia. Sin embargo, resulta llamativo que, entre el grupo de diarios que dieron mayor énfasis y cobertura a los sucesos desfavorables: La Tercera, La Hora, HoyxHoy y el diario Publimetro, a excepción del primero, todos los otros diarios son diarios de distribución gratuita.

En oposición a lo anterior, el diario La Cuarta, El Mercurio y Las Últimas Noticias, publicaron mayor cantidad de noticias en el hito neutro y favorable. Entregando mayor énfasis, al menos para el caso de Nabila Rifo, en los hitos donde se señala al responsable de la agresión (Hito 2) y su consecuencia penal (Hito 3).

\section{Sobre-exposición de la víctima}

El $59 \%$ de las fotografías en portada pertenecían a la víctima, este elemento permite evidenciar que la prensa chilena no respeta el anonimato de las víctimas y, por el contrario, las sobreexpone. Este encuadre gráfico para casos de violencia contra la mujer vulnera el derecho de la privacidad e intimidad de la víctima y puede desvirtuar la comprensión de los hechos interpretándose como un caso aislado, un hecho esporádico.

\section{Contenido:}

\section{Encabezados}

Se cuantificó todas las palabras de los encabezados. A continuación se destacan los 10 conceptos con mayor frecuencia por hito:

Tabla 3.Términos reiterados en encabezados

\begin{tabular}{|l|l|l|l|l|l|l|l|}
\hline \multicolumn{2}{|l|}{ HITO 1} & \multicolumn{2}{|c|}{ HITO 2 } & \multicolumn{2}{|c|}{ HITO 3 } & \multicolumn{2}{c|}{ HITO 4 } \\
\hline 16 & ataque & 20 & Nabila & 5 & Nabila & 5 & Suprema \\
\hline 15 & Coyhaique & 12 & Rifo & 1 & Rifo & & Nabila \\
\hline
\end{tabular}




\begin{tabular}{|r|l|r|l|l|l|l|l|}
\hline 14 & mujer & 9 & juicio & 2 & Ortega & 2 & Corte \\
\hline 13 & Nabila & 9 & ataque & 9 & Mauricio & 1 & condena \\
\hline 12 & agresión & 8 & pareja & 7 & ataque & 8 & Ortega \\
\hline 10 & joven & 7 & Ortega & 7 & juicio & 8 & Rifo \\
\hline 7 & fiscalía & 7 & único & 6 & Coyhaique & 7 & Caso Nabila \\
\hline 7 & Bachelet & 6 & imputado & 6 & imputado & 6 & Mauricio \\
\hline 7 & Rifo & 4 & víctima & 5 & Culpable & 6 & Pena \\
\hline & & & & & & & \\
\hline
\end{tabular}

Fuente: Elaboración propia

\section{Nombre de la víctima}

El nombre y apellido de la víctima (Nabila Rifo), en los cuatro hitos lidera en frecuencia. Lo que demuestra que el encuadre de la prensa chilena, en primer lugar da a conocer el nombre completo de la víctima y, en segundo, focaliza la noticia desde la figura de la víctima, hecho que queda demostrado en el Hito 4, donde ya se nominalizan los hechos bajo el título de Caso Nabila.

\section{Escaso encuadre de la condena}

En el Hito 3 y el Hito 4, se entrega la condena del agresor, no obstante en el último lugar se encuentra: veredicto, culpable, rebaja y pena. En otras palabras, la prensa chilena le otorga 


\section{Yanira Olivares Ramírez.}

una menor presencia a los conceptos que contextualizarían la noticia desde la condena para el victimario. Su menor frecuencia entrega una menor relevancia al castigo penal que tiene un delito como éste.

\section{Relevancia a detalles morbosos}

Por temas de espacio, la tabla de términos no se expone de forma completa. No obstante cabe destacar que en el Hito 1, es posible observar una gran frecuencia de otros conceptos como ojos, sacó, perdió, oculares y globos. Un grupo de palabras que hace referencia a detalles morbosos sobre la agresión cometida en contra de Nabila Rifo, siendo posible inferir que, la prensa chilena, al momento de entregar las primeras informaciones sobre el suceso colocó énfasis en los elementos crudos del caso.

\section{Fotografías e infografías}

Tabla 4. Resultados de fotografías e infografías

\begin{tabular}{|l|c|c|c|c|c|}
\hline HITOS & $\begin{array}{c}\text { HITO } \\
1\end{array}$ & $\begin{array}{c}\text { HITO } \\
2\end{array}$ & $\begin{array}{c}\text { HITO } \\
3\end{array}$ & $\begin{array}{c}\text { HITO } \\
4\end{array}$ & TOTAL \\
\hline Incluye fotografías & 27 & 17 & 19 & 21 & 84 \\
\hline Incluye infografías & 0 & 1 & 1 & 0 & 2 \\
\hline Es una imagen de archivo & 11 & 2 & 6 & 10 & 29 \\
\hline Es una imagen actual & 19 & 15 & 15 & 12 & 61 \\
\hline Es una imagen informativa & 26 & 17 & 19 & 20 & 82 \\
\hline $\begin{array}{l}\text { La imagen pertenece a la } \\
\text { víctima }\end{array}$ & 7 & 15 & 9 & 11 & 42 \\
\hline $\begin{array}{l}\text { La imagen pertenece al } \\
\text { agresor }\end{array}$ & 8 & 4 & 9 & 5 & 26 \\
\hline
\end{tabular}


Yanira Olivares Ramírez.

\begin{tabular}{|l|l|l|l|l|l|}
\hline El pie de foto es informativo & 18 & 7 & 12 & 15 & 52 \\
\hline
\end{tabular}

Fuente: Elaboración propia

\section{Sobre-exposición de la víctima}

Nuevamente se evidencia una gran presencia de material gráfico sobre la víctima. Al sobreexponer a Nabila Rifo, se individualiza un problema social y se entrega un énfasis en la figura de mujer vulnerable y víctima, mostrándola, en algunas ocasiones, siendo asistida por personal de gendarmería o llorando.

\section{La ciudadanía}

Cabe descartar la presencia de fotografías donde se muestran manifestaciones ciudadanas, concentradas principalmente en el Hito 1 como en el Hito 4, ambos sucesos desfavorables para la víctima. Por ello resulta relevante que la prensa haya incorporado y visibilizado la opinión ciudadana, en donde se rechaza la vulneración de los derechos de la mujer.

\section{Fotografías sin contexto}

Teniendo en cuenta que el pie en las fotografías es un elemento que permite contextualizar la imagen, resulta paradójico que las fotografías que tuvieron menos pie de foto informativo, corresponden al material gráfico utilizado en los dos primeros hitos. Teniendo en cuenta que al ser las primeras fotografías del caso el lector tiene menor conocimiento o cercanía con el suceso, deberían ser las que incorporaran un mayor número de información con el fin de contextualizar y encuadrar de mejor forma la noticia.

\section{Cuerpo del texto}

Tabla 5. Resultados del cuerpo del texto

\begin{tabular}{|l|c|c|c|c|c|}
\hline HITOS & HITO & HITO & HITO & HITO & TOTAL \\
& 1 & 2 & 3 & 4 & \\
\hline
\end{tabular}




\begin{tabular}{|c|c|c|c|c|c|}
\hline La protagonista del texto es la víctima & 6 & 11 & 6 & 5 & 28 \\
\hline El protagonista del texto es el agresor & 2 & 1 & 1 & 2 & 6 \\
\hline Los protagonistas del texto son las fuentes & 19 & 8 & 11 & 15 & 53 \\
\hline Se respeta el anonimato de la víctima & 0 & 0 & 0 & 0 & 0 \\
\hline Se respeta la presunción de inocencia del agresor & 26 & 18 & 6 & 0 & 50 \\
\hline $\begin{array}{l}\text { Se hace referencia a otros tipos de violencia que no } \\
\text { sea la física }\end{array}$ & 2 & 7 & 0 & 0 & 9 \\
\hline Caracteriza al agresor por su comportamiento & 2 & 7 & 2 & 0 & 11 \\
\hline Se presenta la noticia como hecho social & 8 & 4 & 3 & 3 & 18 \\
\hline Se presenta la noticia como hecho aislado & 20 & 17 & 16 & 19 & 72 \\
\hline El texto se centra en la exposición de los hechos & 24 & 18 & 19 & 21 & 82 \\
\hline El texto se centra en la descripción del crimen & 4 & 2 & 0 & 1 & 7 \\
\hline Reitera detalles escabrosos o morbosos & 4 & 0 & 2 & 0 & 6 \\
\hline Se incluye información de ayuda a las víctimas & 0 & 1 & 0 & 0 & 1 \\
\hline
\end{tabular}

Fuente: Elaboración propia

\section{Protagonistas de la información}

Se entrega un mayor protagonismo a las fuentes, lo que sugiere un tratamiento adecuado, ya que entrega prioridad a fuentes especializadas y que entregan información objetiva, lo que otorga un gran nivel en la calidad de la información. 


\section{Escasa información educativa}

Se identificó una escasa presencia de información de ayuda a las víctimas (teléfonos o redes de apoyo), caracterización del comportamiento del agresor y referencia a otros tipos de violencia que no sea solo física. Los especialistas recomiendan la incorporación de estos elementos, con el fin de entregar las herramientas para reconocer el comportamiento de un agresor, fomentar las denuncias e idealmente prevenir situaciones de extrema violencia hacia la mujer. Por ende, la ausencia de estos contenidos evidencia que el tratamiento informativo en la prensa chilena no asumió, en el caso de Nabila Rifo, ni un rol educativo ni una perspectiva de género.

\section{Anonimato de la víctima}

Cabe destacar que en ningún momento la prensa chilena respeto el anonimato de la víctima. Es decir, que los medios en Chile, al tratar casos de violencia contra la mujer acostumbran exponer de inmediato a las víctimas. Mientras que en otros países esta práctica periodística es sancionada ${ }^{5}$.

\section{Ausencia de contexto}

Los hechos fueron presentados bajo un contexto aislado, es decir no se situó como un hecho social. Además, las escasas noticias en donde se encuadraba el suceso como un hecho social no incorporaban información suficiente que complejizara la violencia contra la mujer como un acto incitado por los roles de género.

\section{Reiteración de detalles morbosos y escabrosos}

Todavía se presentan noticias en donde se observan detalles del crimen y la reiteración de detalles morbosos y escabrosos. Elementos identificados mayormente en el Hito 1, los que colocaban énfasis en la extracción de sus ojos. Esta postura más sensacionalista que informativa, genera y promueve el morbo en las audiencias y, además expone a Nabila a una segunda

\footnotetext{
${ }^{5}$ Por ejemplo en Madrid, existe la Ley de Medidas de Protección Integral contra la violencia de género, la que entrega una protección reforzada a la intimidad de las víctimas. Las multas a los medios, por exponer el nombre o alguna imagen de la víctima llegan a los 25.000 euros.
} 
victimización, ya que se evidencia una insensibilidad periodística al momento de exhibir la información recogida.

\section{Lenguaje:}

Concepto de violencia

Figura 1. Nominalización del caso

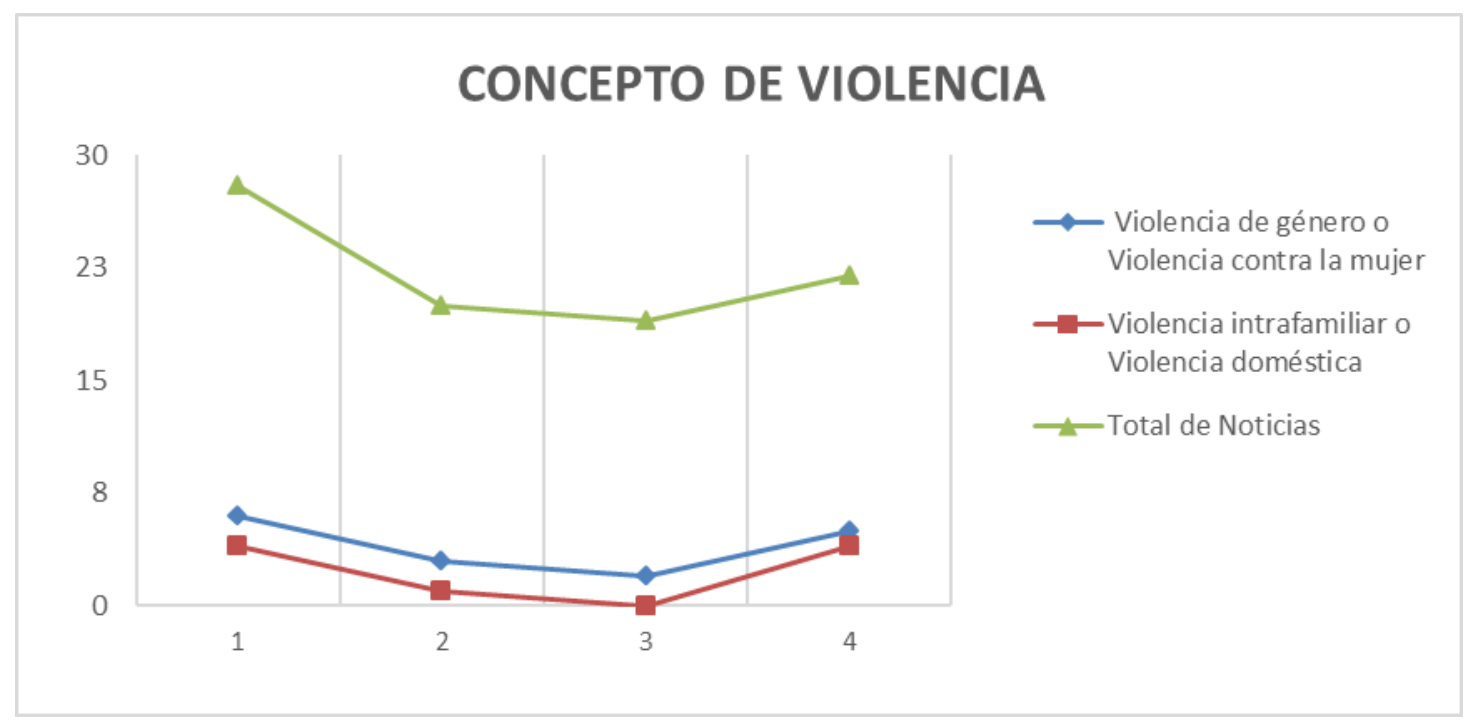

1. Fuente: Elaboración propia

Los conceptos de violencia de género (VG) y violencia contra la mujer ( $\mathrm{VCM}$ ) fueron más utilizados que violencia intrafamiliar (VIF) o violencia doméstica (VD) -16 versus 9 - al momento de informar sobre el caso de Nabila Rifo. No obstante, cabe destacar que además de su escasa diferencia, el grupo editorial Copesa posicionó mayormente los conceptos de VG y VCM, mientras que el grupo editorial El Mercurio S.A. utilizó principalmente los conceptos de VIF y $\mathrm{VD}$, mientras que Metro International utilizó ambos grupos de forma equitativa. De este modo, se evidencia que el grupo editorial Copesa avanza en el tema, ya que incorpora en el tratamiento informativo los términos que sugieren los especialistas VG y VCM, a diferencia de los otros grupos editoriales.

\section{Ausencias}




\section{Yanira Olivares Ramírez.}

No obstante, las ausencias también son significativas al momento de encuadrar un hecho noticioso. De este modo, si se considera que de un total de 89 noticias solo 25 de ellas incorporaron conceptos como estos, es posible inferir que la prensa chilena no encuadró las noticias del caso bajo la preponderancia de ningún concepto de violencia. Probablemente, su escasez remita a dificultades o confusiones que aún genera la tipificación de estos hechos bajo un delito (VIF) o el otro (VG), y por lo tanto los medios prefieren omitirlo ${ }^{6}$.

En la misma línea, se generó subcategorías ${ }^{7}$ con una propuesta de conceptos inadecuados que desvirtuaron el tratamiento informativo en las noticias sobre Nabila Rifo:

\section{Tabla 6. Términos inadecuados}

\begin{tabular}{|c|c|c|c|c|c|c|}
\hline \multicolumn{2}{|l|}{ HITOS } & $\begin{array}{l}\text { HITO } \\
1\end{array}$ & $\begin{array}{l}\text { HITO } \\
2\end{array}$ & $\begin{array}{l}\text { HITO } \\
3\end{array}$ & $\begin{array}{l}\text { HITO } \\
4\end{array}$ & TOTAL \\
\hline \multirow{5}{*}{$\begin{array}{l}\text { MÓVILES Y JUSTIFICACIÓN } \\
\text { (Ej: por infiel, por celos, } \\
\text { respondiendo a sus bajos } \\
\text { instintos, porque lo iba a dejar) }\end{array}$} & alcohol & 6 & 5 & 1 & 0 & 12 \\
\hline & drogas & 0 & 3 & 0 & 0 & 3 \\
\hline & pechos & 0 & 1 & 0 & 0 & 1 \\
\hline & bestialmente & 0 & 0 & 1 & 0 & 1 \\
\hline & salvajemente & 4 & 1 & 2 & 1 & 8 \\
\hline $\begin{array}{l}\text { PATOLOGIZAR AL } \\
\text { AGRESOR } \\
\text { (Ej: enfermos, psicópatas, } \\
\text { celópata) }\end{array}$ & & & & & & 0 \\
\hline MUJER VULNERABLE & joven & 12 & 1 & 0 & 0 & 13 \\
\hline
\end{tabular}

${ }^{6}$ Las estadísticas internacionales y nacionales, demuestran que las principales víctimas de violencia intrafamiliar son mujeres. En Chile el Ministerio del Interior y seguridad pública, en sus cifras sobre VIF con lesiones menos graves, graves o gravísimas arroja que un $78 \%$ de las víctimas son mujeres. Asimismo, el $80 \%$ de las víctimas de VIF con lesiones psicológicas o lesiones leves son mujeres (2015). Por lo tanto la "violencia intrafamiliar", en muchas ocasiones, invisibiliza que el problema de fondo radica en una violencia de género.

${ }^{7}$ Por falta de espacio no se expondrá las definiciones de cada categoría generada, sin embargo esta se encuentra disponible en la tesis, la que está disponible en el repositorio de la USACH. 


\section{Yanira Olivares Ramírez.}

\begin{tabular}{|l|l|c|c|c|c|c|}
\hline \multirow{2}{*}{$\begin{array}{l}\text { (Ej: mujer sola, débil, joven, } \\
\text { bella, mujer en peligro) }\end{array}$} & fiesta & 2 & 2 & 2 & 0 & 6 \\
\cline { 2 - 7 } & prostitución & 0 & 1 & 0 & 0 & 1 \\
\hline $\begin{array}{l}\text { NATURALIZACIÓN } \\
\text { (Ej: crimen por honor, crimen } \\
\text { pasional, por razones } \\
\text { sentimentales) }\end{array}$ & & & & & & \\
\hline $\begin{array}{l}\text { VERBOS INTRANSITIVOS } \\
\text { (Ej: mujer muere, mujer murió) }\end{array}$ & perdió sus ojos & 4 & 0 & 0 & 0 & 4 \\
\hline \multirow{2}{*}{$\begin{array}{l}\text { DESAPAREZCA EL } \\
\text { AGRESOR } \\
\text { (Ej: violador serial, maldito, } \\
\text { sátiro) }\end{array}$} & salvaje & 1 & 0 & 0 & 0 & 1 \\
\cline { 2 - 7 } & mergúmeno & 1 & 0 & 0 & 0 & 1 \\
\hline & troglodita & 1 & 0 & 0 & 0 & 1 \\
\hline $\begin{array}{l}\text { AGRESOR CON OTROS } \\
\text { DELITOS } \\
\text { (Ej: narcotráfico, abuso sexual, } \\
\text { robos, violación de morada) }\end{array}$ & intrafamiliar & 6 & 0 & 2 & 0 & 8 \\
\hline $\begin{array}{l}\text { ERROR INFORMATIVO } \\
\text { violencia }\end{array}$ & error en el nombre & 0 & 1 & 0 & 0 & 1 \\
\hline
\end{tabular}

Fuente: Elaboración propia

\section{Grupos sin términos encontrados}

No se identificó ningún término en el grupo de Patologizar al agresor y Naturalización. La ausencia de términos en estos grupos, permite deducir que existe un mayor conocimiento y sensibilidad por parte de los profesionales, es decir están más familiarizados frente a estos errores, lo que significa un avance.

\section{Términos inadecuados}




\section{Yanira Olivares Ramírez.}

No obstante, se cuantificó 63 veces el uso de algún término inadecuado perteneciente a los grupos propuestos, lo que demuestra que aún existe un escaso conocimiento sobre otros tipos de conceptos que desvirtúan un tratamiento adecuado para los casos de VCM.

\section{Hito 1, el más inadecuado}

La concentración de términos inadecuados en el Hito 1, pertenecen en su mayoría a las subcategorías de móviles y justificación y mujer vulnerable. Estos resultados son preocupantes, ya que los términos del primer grupo disminuyen la responsabilidad del agresor, incorporando causas que justifican su actuar (alcohol, drogas, pechos, etc.), y el segundo grupo de términos, focaliza la responsabilidad en la víctima, tanto por sus características (joven) como por el contexto en el que se encontraba (fiesta, madrugada). Esto demuestra, que la prensa en Chile sigue reproduciendo roles de género, culpabilizando a las mujeres por sus agresiones $\mathrm{y}$, disminuyendo la responsabilidad de los agresores.

\section{Diferencias por grupo editorial}

Cabe destacar que los diarios que incorporaron en mayor número algún término inadecuado son el diario La Cuarta y el diario La Tercera, ambos medios pertenecientes al grupo editorial Copesa.

\section{Fuentes:}

Tabla 7. Resultados de las fuentes

\begin{tabular}{|l|c|c|c|c|c|}
\hline HITOS & $\begin{array}{c}\text { HITO } \\
1\end{array}$ & $\begin{array}{c}\text { HITO } \\
2\end{array}$ & $\begin{array}{c}\text { HITO } \\
3\end{array}$ & $\begin{array}{c}\text { HITO } \\
4\end{array}$ & TOTAL \\
\hline Incluye el testimonio de familiares & 5 & 0 & 1 & 1 & 7 \\
\hline Incluye el testimonio de vecinos & 13 & 1 & 2 & 0 & 16 \\
\hline Incluye fuentes de carácter oficial & 28 & 16 & 18 & 19 & 81 \\
\hline
\end{tabular}


Yanira Olivares Ramírez.

\begin{tabular}{|l|c|c|c|c|c|}
\hline $\begin{array}{l}\text { Incluye el testimonio de otras víctimas de violencia de } \\
\text { género }\end{array}$ & 1 & 0 & 0 & 0 & 1 \\
\hline Incluye testimonios que inculpen a la mujer & 0 & 0 & 1 & 0 & 1 \\
\hline Incluye testimonios que exculpen al agresor & 1 & 2 & 4 & 0 & 7 \\
\hline
\end{tabular}

Fuente: Elaboración propia

\section{Diversidad de voces}

Fue posible constatar una gran presencia de fuentes oficiales de diversas áreas de especialización (gobierno, salud, judicial, penal, investigaciones). Asimismo, se incluyó dichos de vecinos, quienes aportaban información que remitía exclusivamente a los hechos ocurridos y no a conjeturas u opiniones sobre la víctima o victimario. También se recurrió a los familiares, quienes entregaron una descripción tanto de la víctima como del victimario -familiares respectivos a cada individuo-, si bien esta información resulta ser menos objetiva, su presencia fue escasa y de menor relevancia.

\section{Errores}

Se observó sesgos informativos, incorporando el testimonio de una cercana al victimario, quien -en un programa de televisión- responsabiliza a Nabila por lo sucedido. En la misma línea, se observan testimonios que exculpan al agresor, los que remiten tanto a su abogado, un bioquímico de la defensa, un testigo y al sobrino de Mauricio Ortega.

La incorporación de estas fuentes, se encuentran con mayor preponderancia en el único hito donde la víctima es favorecida, Hito 3-Veredicto en donde se declara culpable a Mauricio. Estos elementos sugieren un encuadre inadecuado sobre el caso, ya que insinúa dudas sobre el fallo y la responsabilidad del agresor, lo que beneficia al agresor y desfavorece principalmente a la víctima.

\section{Fuentes especializadas en temas de género}




\section{Yanira Olivares Ramírez.}

Se identificó líderes de opinión condenando y rechazando la agresión, incorporación favorable ya que, al ser fuentes conocidas, son sujetos que generan un mayor impacto y credibilidad en las audiencias.

Sin embargo, la incorporación de fuentes expertas en temas de género y violencia fue escasa. Esta práctica periodística dificulta la formación de una sociedad con una opinión pública crítica, reflexiva y con perspectiva de género frente a casos como estos.

\section{Conclusiones}

A partir del análisis a la prensa chilena, representada por ocho diarios, se constató un gran interés por visibilizar y mantener en la agenda mediática un caso de violencia contra la mujer, como el de Nabila Rifo. No obstante, el tratamiento periodístico sigue siendo cómplice de la violencia contra las mujeres, ya que generan encuadres en donde: ponen énfasis en los contextos negativos para las víctimas, sobreexponen material gráfico de la víctima, presentan escaso hincapié en la condena o rechazo a los actos del agresor, no contextualizan la situación como un problema social, reiteran detalles morbosos, no incorporan términos como violencia de género o violencia contra la mujer, no entregan información educativa para concientizar a la población, utilizan términos inadecuados y no recurren a fuentes especializadas en violencia de género.

Al carecer de un abordaje adecuado, sustancial y con perspectiva de género no contribuyen a erradicar la violencia contra la mujer, sino que, muy por el contrario, originan un segundo espacio de violencia para las víctimas, y al mismo tiempo, continúan construyendo representaciones simbólicas que han perpetuado históricamente la violación de los derechos fundamentales de las mujeres.

Entendiendo que los medios de comunicación son instituciones culturales que construyen y producen determinadas formas de ver el mundo y valorarlo, indudablemente resulta urgente que la agenda mediática cumpla su rol social, y colabore en su erradicación.

A continuación podría emitir una serie de recomendaciones para un tratamiento adecuado, sin embargo resulta una tarea infructuosa, ya que actualmente existen innumerables textos sobre esas temáticas y aún así los medios de comunicación tradicionales no las incorporan. Esto permite suponer, que los medios de comunicación no están interesados en el desarrollo de un país libre de violencia ni en revertir las desigualdades de género. 


\section{Yanira Olivares Ramírez.}

En conclusión, como lo plantea Rita Segato, "no podemos tener las mismas aspiraciones que los hombres dentro del orden patriarcal, porque si no vamos reproduciendo lo mismo pero con otros cuerpos, tenemos que conseguir imaginar un mundo nuevo, un mundo realmente distinto" (2019). Un nuevo mundo mediático que no haga eco de los frame ni de las representaciones simbólicas que hemos heredado. Un espacio público que reconfiguraremos de forma colectiva, donde la lucha sea principalmente contra las masculinidades hegemónicas que destruyen, matan y violan a mujeres, niñas, niños, gais, lesbianas, transexuales, transgéneros y hasta a los mismos "hombres". De nosotras y nosotros depende cambiar nuestra historia y mostrarle a los medios hegemónicos que hoy, más que nunca, entendemos aquel concepto llamado APROPIACIÓN.

\section{Bibliografía}

Ananías, C. y Vergara, K. (2016). Tratamiento informativo del feminicidio en los medios de comunicación digitales chilenos en marzo de 2016: Una aproximación al horizonte actual desde la perspectiva de género. En Comunicación y Medios, 25 (34), 52 - 69.

Angélico, R., Dikenstein, V., Fischberg, S. y Maffeo, F. (2014). El feminicidio y la violencia de género en la prensa argentina: un análisis de voces, relatos y actores. En Universitas Humanística, 78, 281-303. Recuperado desde: http://dx.doi.org/10.11144/Javeriana.UH78.fvgp

Aruguete, N. (2011). Framing. La Perspectiva de las noticias. La Trama de la comunicación, Volumen 15, pp. 67-80. ISSN: 1668-5628.Rosario: UNR.

Beauvoir, S. (1949). El segundo sexo. Paris: Editorial Gallimard.

Bourdieu, P. (2000). La dominación masculina. Barcelona: Editorial Anagrama.

Global Media Monitoring Project (2010). GMMP 2010 Reports. Recuperado desde: http://whomakesthenews.org/gmmp/gmmp-reports/gmmp-2010-reports

International Women's Media Foundation. IWMF. (2011).Global Report on the status of women the News Media. Washington: USA Recuperado en: https://www.iwmf.org/wpcontent/uploads/2013/09/IWMF-Global-Report-Summary.pdf ISBN 9780-615-452708

Jiménez, M. (coord.). (2005). Caras de la violencia familiar. Segundo Seminario sobre Violencia Familiar en el Distrito Federal". Universidad Autónoma de México y Dirección General de Equidad y Desarrollo Social, Colección La ciudad. México.

Krippendorff, K. (1990). Metodología de análisis de contenido. Teoría y Práctica. Barcelona: Paidós. 
Lagunas, C. y Lencina, K. (2010). El registro periodístico y los paradigmas culturales aceptados: La violencia sobre la mujer. En La aljaba, 14, 121-134. Recuperado desde: http://www.scielo.org.ar/scielo.php?script=sci_arttext\&pid=S166957042010000100007 \&lng=es\&tlng=es

Lippmann, W. (1992). La opinión pública. Madrid: Langre.

Morales, O. y González, C. (2007). Consideraciones discursivas sobre el género en el discurso académico e institucional: ¿dónde está ella?. En Educere, 11(38), 433-453. Recuperado desde: http://www.scielo.org.ve/scielo.php?script=sci_arttext\&pid=S131649102007000300010\&lng=es\&tlng=es

Observatorio de Género y Equidad. OGE. (2016). Sin mujeres no hay periodismo. Recuperado desde: http://oge.cl/sin-mujeres-no-hay-periodismo/

Pérez, E. (2001). La violencia contra la mujer en la prensa. En Revista Latina de Comunicación Social, 44. Recuperado desde: http://www.ull.es/publicaciones/latina/2001/latina44septiembre/4408perezsalicio. htm

Rico, N. (1996).Violencia de género: un problema de derechos humanos. Serie Mujer y Desarrollo, CEPAL.

Segato, R. (2019). Conferencia Patriarcado y violencia hoy. Santiago: Universidad de Chile.

Scott, J. (1996). Género: una categoría útil para el análisis histórico. En Lamas M.(comp.) El género: construcción cultural de la diferencia sexual. México: PUEG.

Thompson, J. (1998). Los media y la modernidad. Una teoría de los medios de comunicación. Barcelona: Paidos. 